\title{
ARTICLE \\ Rifampicin induces clathrin-dependent endocytosis and ubiquitin-proteasome degradation of MRP2 via oxidative stress-activated PKC-ERK/JNK/p38 and PI3K signaling pathways in HepG2 cells
}

\author{
Bao-yan $\mathrm{Xu}^{1}$, Xu-dong Tang ${ }^{2}$, Jing Chen ${ }^{2}$, Hong-bo $\mathrm{Wu}^{2}$, Wen-sheng Chen ${ }^{2}$ and Lei Chen ${ }^{2}$
}

It was reported that antituberculosis medicines could induce liver damage via oxidative stress. In this study, we investigated the effects of rifampicin (RFP) on the membrane expression of multidrug resistance-associated protein 2 (MRP2) and the relationship between oxidative stress and RFP-induced endocytosis of MRP2 in HepG2 cells. We found that RFP (12.5-50 $\mu$ M) dose-dependently decreased the expression and membrane localization of MRP2 in HepG2 cells without changing the messenger RNA level. RFP $(50 \mu \mathrm{M})$ induced oxidative stress responses that further activated the PKC-ERK/JNK/p38 (protein kinase C-extracellular signalregulated kinase/c-JUN N-terminal kinase/p38) and PI3K (phosphoinositide 3-kinase) signaling pathways in HepG2 cells. Pretreatment with glutathione reduced ethyl ester $(2 \mathrm{mM})$ not only reversed the changes in oxidative stress indicators and signaling molecules but also diminished RFP-induced reduction in green fluorescence intensity of MRP2. We conducted coimmunoprecipitation assays and revealed that a direct interaction existed among MRP2, clathrin, and adaptor protein 2 (AP2) in HepG2 cells, and their expression was clearly affected by the changes in intracellular redox levels. Knockdown of clathrin or AP2 with small interfering RNA attenuated RFP-induced decreases of membrane and total MRP2. We further demonstrated that RFP markedly increased the ubiquitin-proteasome degradation of MRP2 in HepG2 cells, which was mediated by the E3 ubiquitin ligase GP78, but not HRD1 or TEB4. In conclusion, this study demonstrates that RFP-induced oxidative stress activates the PKC-ERK/JNK/ p38 and PI3K signaling pathways that leads to clathrin-dependent endocytosis and ubiquitination of MRP2 in HepG2 cells, which provides new insight into the mechanism of RFP-induced cholestasis.

Keywords: rifampicin; cholestasis; MRP2; oxidative stress; PKC-ERK/JNK/p38; PI3K; endocytosis; cholestasis; HepG2 cells

Acta Pharmacologica Sinica (2020) 41:56-64; https://doi.org/10.1038/s41401-019-0266-0

\section{INTRODUCTION}

Oxidation and antioxidant systems exist in a dynamic equilibrium under normal conditions in organisms. However, upon exposure to harmful factors or under pathological conditions, organisms produce excessive reactive oxygen species (ROS) that overwhelm the elimination capacity of the antioxidant systems, which induces oxidative stress [1]. Oxidative stress can directly lead to tissue injury via lipid peroxidation and can activate many cytokines through the protein kinase $C$ (PKC) or mitogen-activated protein kinase (MAPK) signaling pathway, which further promotes the development of tissue damage. It has been discovered that antituberculosis drugs induce liver damage via oxidative stress associated with increases in cellular oxidants, such as the lipid peroxidation product malondialdehyde (MDA), and with consumption of antioxidants, including superoxide dismutase (SOD) and glutathione (GSH) [1-5].

Rifampicin (RFP) is a first-line antituberculosis drug recommended by the World Health Organization that is infamous for its hepatotoxicity, which is the main side effect of tuberculosis treatment and the most critical factor restricting the clinical application of this drug $[6,7]$. RFP causes liver damage mainly by cholestasis, with an obvious increase in serum bilirubin as the major clinical manifestation. This effect is closely associated with hepatocyte canalicular transporters, such as multidrug resistanceassociated protein 2 (MRP2), which localizes to the hepatocyte apical membrane $[8,9]$. Previous studies have shown that RFP, an activator of pregnane $X$ receptor, promotes the excretion of bile salts by upregulating the expression of MRP2 in hepatocytes. However, this finding is inconsistent with the clinical phenomenon of biliary siltation caused by RFP [10]. Interestingly, studies on rat models of cholestasis have shown that impaired MRP2-mediated transport coincides with strongly decreased MRP2 protein levels and endocytic retrieval of MRP2 without any significant changes in MRP2 messenger RNA (mRNA) levels [11]. Disrupted canalicular localization and decreased MRP2 protein expression without changes in MRP2 mRNA expression have also been observed in patients with chronic cholestatic disorder and hepatic failure, suggesting that post-transcriptional regulation of MRP2, such as

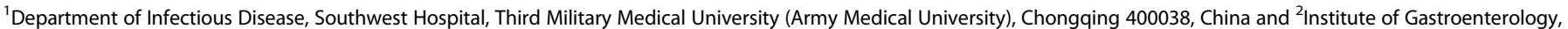
Southwest Hospital, Third Military Medical University (Army Medical University), Chongqing 400038, China

Correspondence: Lei Chen (13883895566@163.com)

Received: 18 October 2018 Accepted: 30 May 2019

Published online: 17 July 2019 
Rifampicin induced ubiquitination of MRP2 in HepG2 cells BY Xu et al.

redistribution of MRP2 from the canalicular membrane into the cytosol (i.e., endocytic retrieval) followed by ubiquitination, may be considered a decisive step contributing to decreased bile flow $[12,13]$. Therefore, we speculated that RFP-induced cholestasis may be associated with endocytosis of MRP2 and that this process is perhaps the critical reason for RFP-induced hepatotoxicity. However, there have been no relevant reports until now.

Studies have shown that oxidative stress is sometimes accompanied by cholestasis and may be a key factor for endocytosis of envelope proteins. Sekine and Horie [14] reported that MRP2 was internalized when acute oxidative stress arose in rat livers and returned to the canalicular membrane after replenishment of intracellular GSH, which was related to the redox-sensitive balance of protein kinase $A$ (PKA)/PKC activation. In the present study, we studied the effects of RFP on MRP2 in HepG2 human hepatic epithelial cells and explored the underlying mechanisms, and we found that RFP initiated endocytosis and ubiquitin-proteasome degradation of MRP2 on the hepatocyte membrane via oxidative stress. Our findings lay a foundation for further research on and prevention of RFP-induced cholestasis.

\section{MATERIALS AND METHODS}

Cell culture and processing

HepG2 cells (Shanghai Institute of Biochemistry and Cell Biology, Shanghai, China) were incubated in high-glucose Dulbecco's modified Eagle's medium (Gibco, CA, USA) supplemented with $10 \%$ fetal bovine serum (Invitrogen, CA, USA), $100 \mathrm{U} / \mathrm{mL}$ penicillin, $100 \mu \mathrm{g} / \mathrm{mL}$ streptomycin, and $0.25 \mathrm{ng} / \mathrm{mL}$ amphotericin B (Invitrogen, $\mathrm{CA}$, USA) in a humid atmosphere with $5 \% \mathrm{CO}_{2}$ at $37^{\circ} \mathrm{C}$. The medium was changed every $24 \mathrm{~h}$.

Cells were seeded at a density of $2 \times 10^{5}$ cells per well into sixwell plates $24 \mathrm{~h}$ before drug treatments. Glutathione reduced ethyl ester (GSH-MEE), RFP, hydrogen peroxide $\left(\mathrm{H}_{2} \mathrm{O}_{2}\right), \mathrm{MG} 132$, probenecid, and dimethyl sulfoxide (DMSO) were all purchased from Sigma-Aldrich (St. Louis, MO, USA). GSH-MEE (2 mM, dissolved in water) was added to the medium $1 \mathrm{~h}$ before RFP $(12.5,25$, or $50 \mu \mathrm{M}$, dissolved in DMSO) was added. Probenecid $(50 \mu \mathrm{M})$ was used as a positive control for MRP2 inhibition. An equal volume of DMSO was added as the RFP negative control, and $1 \mathrm{mM} \mathrm{H} \mathrm{H}_{2}$ was used as a positive control for oxidative stress. To test whether RFP can degrade MRP2 through the ubiquitin-proteasome pathway, MG132 (5 $\mu \mathrm{M})$ was added to the medium $12 \mathrm{~h}$ before harvesting the cells. The duration of RFP stimulation was $24 \mathrm{~h}$.

\section{Cell transfection}

Small interfering RNAs (siRNAs) for clathrin, adaptor protein 2 (AP2), and GP78 and a negative control siRNA were purchased from Invitrogen. HepG2 cells at approximately $60 \%$ confluence were transiently transfected with the indicated combinations of siRNAs using Lipofectamine 2000 transfection reagent (Invitrogen, (A, USA) in strict accordance with the manufacturer's instructions. Then, $48 \mathrm{~h}$ post transfection, Western blotting was used to detect the transfection efficiency [15].

\section{Measurement of oxidative stress indicators}

The supernatant was collected after HepG2 cells were disrupted by ultrasound for subsequent detection of GSH, SOD, and MDA (Solarbio, Beijing, China) levels with microassay kits as per the manufacturer's directions.

Quantitative reverse transcription-polymerase chain reaction According to the manufacturer's protocols, $1 \mu \mathrm{g}$ of total RNA extracted from HepG2 cells with RNAiso Plus (TaKaRa, Dalian, China) was reverse transcribed with a PrimeScript ${ }^{\mathrm{TM}}$ RT Reagent Kit with gDNA Eraser (TaKaRa, Dalian, China). Real-time quantitative PCR was performed with a SYBR ${ }^{\varpi}$ Premix Ex Taq $^{\text {TM }}$ II Kit
Table 1. Primer sequences used in RT-qPCR

\begin{tabular}{ll}
\hline Name & Sequence \\
\hline MRP2 sense & 5'-AGCACCGACTATCCAGCATC-3' $^{\prime}$ \\
MRP2 antisense & 5'-GAAACCAAAGGCACTCCAGA-3' $^{\prime}$ \\
Clathrin sense & $5^{\prime}$-GGCCCAGATTCTGCCAATTCGTTT-3' \\
Clathrin antisense & $5^{\prime}$-TGATGGCGCTGTCTGCTGAAATTG-3' \\
AP2 sense & $5^{\prime}$-GGTGAACCCCAACGAAGTCT-3' \\
AP2 antisense & $5^{\prime}$-GGGATCGGAATGTTGTCGGT-3' \\
GAPDH sense & $5^{\prime}$-GGGAGCCAAAAGGGTCATCA-3' \\
GAPDH antisense & $5^{\prime}$-TGATGGCATGGACTGTGGTC-3' \\
\hline
\end{tabular}

$R T-q P C R$ quantitative reverse transcription-polymerase chain reaction, $A P 2$ adaptor protein 2, MRP2 multidrug resistance-associated protein 2, GAPDH glyceraldehyde-3-phosphate dehydrogenase

(TaKaRa, Dalian, China) in an Applied Biosystems 7500 Fast realtime PCR system to detect the mRNA levels of specific genes. Glyceraldehyde-3-phosphate dehydrogenase (GAPDH) was used as a reference gene to normalize the data. The primers are described in Table 1.

Immunofluorescence analysis

Immunofluorescence analysis was performed as previously described [16]. Briefly, after the samples were fixed with $4 \%$ paraformaldehyde and blocked with phosphate-buffered saline containing $1 \%$ bovine serum albumin and $0.3 \%$ Triton X-100, the proteins were detected with primary antibodies followed by goat anti-rabbit immunoglobulin G (lgG) Alexa Fluor 488 (1:500 dilution, ab150077, Abcam) or goat anti-rabbit IgG Alexa Fluor 568 (1:500 dilution, ab175471, Abcam) secondary antibodies in the dark for $1 \mathrm{~h}$. The primary antibodies included a rabbit MRP2 polyclonal antibody (1:100, sc-20766, Santa Cruz, CA, USA), a rabbit clathrin heavy-chain monoclonal antibody $(1: 100, \# 4796$, Cell Signaling), and a rabbit AP2a monoclonal antibody (1:100, \#3215, Cell Signaling). The nuclei were stained with Hoechst 33342. Images were obtained with a confocal laser scanning microscope (LSM510, Carl Zeiss, Jena, Germany)

Western blot analysis

Total proteins or membrane proteins were extracted from HepG2 cells with high-efficiency RIPA tissue/cell lysis buffer (R0010, Solarbio) or a Minute ${ }^{\mathrm{TM}}$ Plasma Membrane Protein Isolation Kit (SM-005, Invent Biotechnologies), respectively. The samples $(40 \mu \mathrm{g}$ per well) were separated by $8 \%$ or $10 \%$ sodium dodecyl sulfonatepolyacrylamide gel electrophoresis and then transferred onto polyvinylidene difluoride membranes (Millipore). After blocking with $5 \%(\mathrm{w} / \mathrm{v})$ nonfat milk, the blots were probed with primary antibodies at 1:1000 dilutions. A rabbit MRP2 polyclonal antibody (sc-20766) was purchased from Santa Cruz, and a rabbit PKCa monoclonal antibody (ab32376), a PKC $\varepsilon$ monoclonal antibody (ab124806), a PKC $\delta$ monoclonal antibody (ab182126), an GP78 antibody (ab227450), an anti-SYVN1/HRD1 antibody (ab170901), and an anti-MARCH6/TEB4 antibody (ab183533) were purchased from Abcam. A phospho (p)-PI3K polyclonal antibody (\#4228), a PI3K monoclonal antibody (\#4249), a p-p44/42 MAPK (ERK1/2) monoclonal antibody (\#4370), a p44/42 MAPK (ERK1/2) monoclonal antibody (\#4695), a p-SAPK/JNK antibody (\#9251), a SAPK/ JNK antibody (\#9252), a p-P38 MAPK monoclonal antibody (\#4511), a P38 MAPK antibody (\#9212), a clathrin heavy-chain monoclonal antibody (\#4796), a p-AP2 monoclonal antibody (\#7399), a GAPDH monoclonal antibody (\#2118), a ubiquitin antibody (\#3933) and a $\mathrm{Na}^{+} / \mathrm{K}^{+}$ATPase antibody (\#3010) were all obtained from Cell Signaling. Horseradish peroxidase-conjugated goat anti-rabbit IgG (1:5000, ab6721, Abcam) was used as the 
a

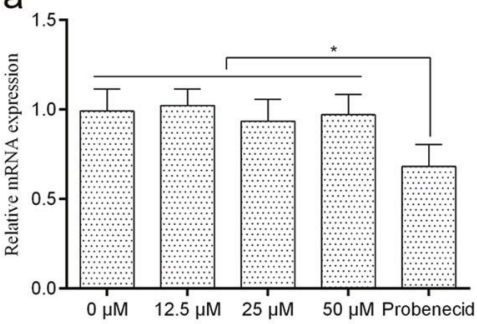

b

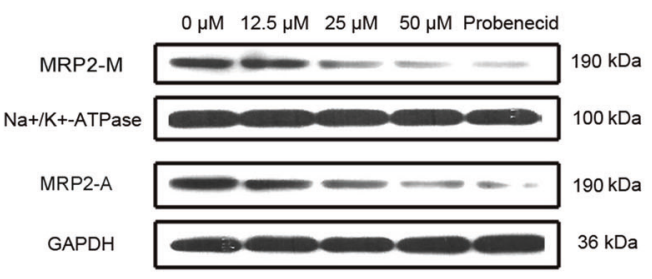

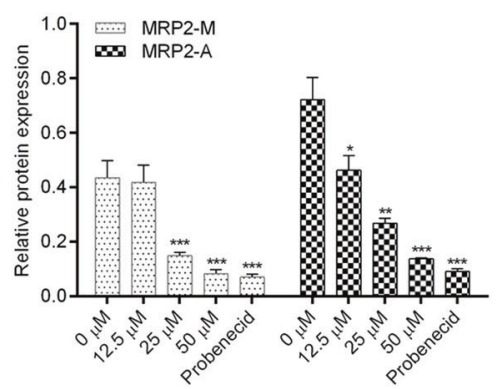

C
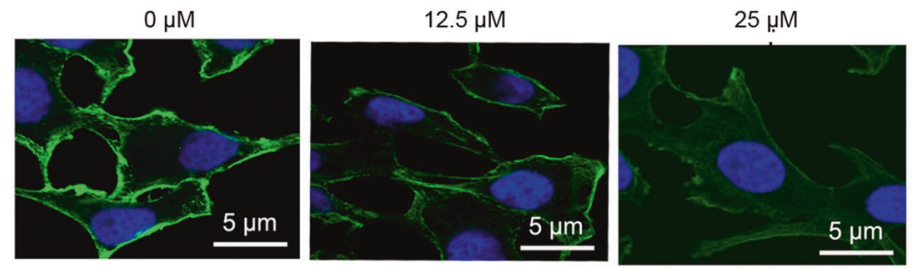

Probenecid
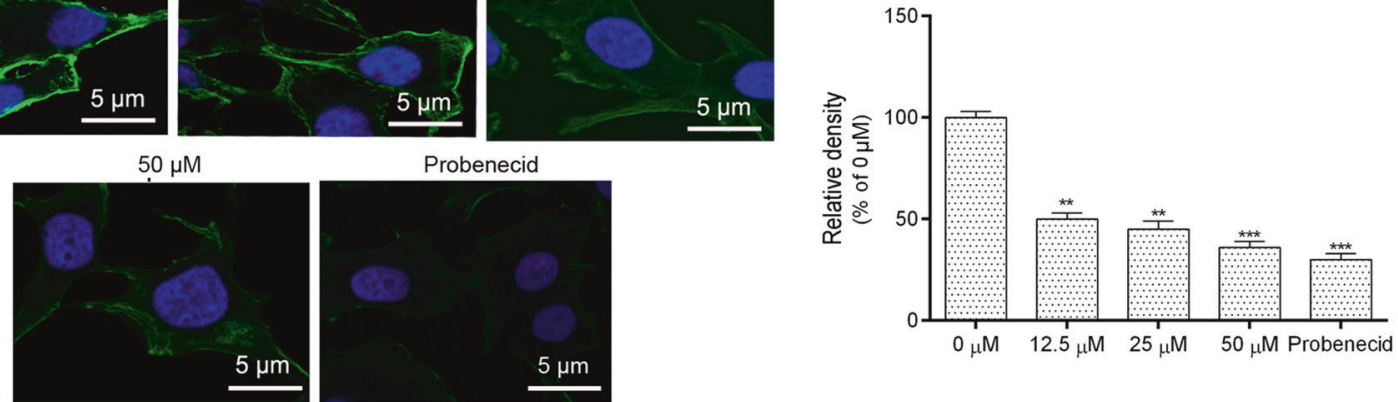

Fig. 1 Effects of rifampicin (RFP) at different doses on multidrug resistance-associated protein 2 (MRP2) expression in HepG2 cells. a The relative messenger RNA (mRNA) expression of MRP2 was detected by quantitative reverse transcription-polymerase chain reaction (qRT-PCR). b Western blotting was used to analyze the protein expression of total MRP2 and membrane MRP2. ImageJ software was used to quantify the relative protein expression. c Localization of MRP2 was detected by confocal laser scanning microscopy. The green fluorescence represents MRP2 protein, and the blue fluorescence represents nuclei. Scale bar, $5 \mu \mathrm{m}$. The fluorescence intensity of MRP2 was measured with Image-Pro Plus software. The values are expressed as the mean \pm SD from three independent experiments $(n=3)$. ${ }^{*} P<0.05,{ }^{* *} P<0.01$ and ${ }^{* * *} P<0.001$ vs. $0 \mu$ M. MRP2-M: MRP2 on the membrane; MRP2-A: total MRP2. Magnification: $\times 400$

secondary antibody. The signals were generated with an Enhanced Chemiluminescence Detection Kit (Beyotime, Shanghai, China) and were detected with an automatic molecular imaging system (Tanon-5500). Densitometric analysis was performed with ImageJ software.

\section{Co-immunoprecipitation assay}

Co-immunoprecipitation (Co-IP) assays were conducted with a Pierce Co-IP Kit (Thermo, 21649) according to the manufacturer's specifications. Briefly, HepG2 cells were lysed with lysis/wash buffer on ice. The supernatant was collected and quantified with a BCA Protein Assay Kit (PC0020, Solarbio). Then, $300 \mu \mathrm{g}$ of lysate was added to a spin column containing a resin slurry that had previously been combined with MRP2 antibody or lgG (as a negative control) and shaken overnight at $4{ }^{\circ} \mathrm{C}$. After elution and centrifugation, the proteins combined with the MRP2 antibody were obtained and analyzed by Western blotting.

Statistical analysis

The data are presented as the mean \pm standard deviation (SD) from three independent experiments. Statistical analysis was carried out with one-way analysis of variance with SPSS 12.0 software. A value of $P<0.05$ was considered to indicate statistical significance.

\section{RESULTS}

Effects of RFP on the expression of MRP2 in HepG2 cells As shown in Fig. 1a, probenecid markedly decreased the MRP2 mRNA levels in the treated groups compared to the $0 \mu \mathrm{M}$ group, while the MRP2 mRNA levels did not change significantly under RFP stimulation at doses of $12.5-50 \mu \mathrm{M}$. However, both probenecid and RFP stimulation reduced membrane MRP2 (MRP2-M) and total MRP2 (MRP2-A) expression (Fig. 1b). Similarly, immunofluorescence analysis revealed that the green fluorescence intensity indicating MRP2 expression on the cell membrane was clearly downregulated under RFP and probenecid stress compared to control conditions (Fig. 1c). These results indicated that RFP regulated the expression of MRP2 at the posttranscriptional level.

RFP decreased the membrane distribution of MRP2 by activating oxidative stress in HepG2 cells

Next, we detected oxidative stress markers, including GSH, MDA, and SOD, in HepG2 cells treated with RFP at a dose of $50 \mu \mathrm{M}$. Compared to those in the control group, the concentrations of GSH (Fig. 2a) and SOD (Fig. 2b) were significantly decreased and those of MDA (Fig. 2c) were markedly increased in the RFP and $\mathrm{H}_{2} \mathrm{O}_{2}$ groups. However, the addition of GSH-MEE reversed the changes in GSH, SOD, and MDA levels, indicating that RFP induced oxidative stress in HepG2 cells. Similarly, HepG2 cells in the GSHMEE pretreatment group showed stronger green fluorescence of MRP2 on the membrane than those in the RFP and $\mathrm{H}_{2} \mathrm{O}_{2}$ groups (Fig. 2d). These results demonstrated that RFP decreased the membrane distribution of MRP2 via oxidative stress.

RFP activated the PKC-ERK/JNK/p38 and PI3K signaling pathways via oxidative stress

After treatment with RFP and $\mathrm{H}_{2} \mathrm{O}_{2}$, the expression of PKCa, PKC and PKCE (Fig. 3a, b) and the phosphorylation levels of JNK, P38, and ERK (Fig. 3a, c) were clearly upregulated, while p-PI3K was significantly downregulated, in the treated groups compared to the control group. However, GSH-MEE successfully inhibited the decrease in p-PI3K and the activation of PKCa, PKC, PKC $\varepsilon, p-J N K$, p-P38, and $p$-ERK induced by RFP, indicating that RFP activated the PKC-ERK/JNK/p38 and PI3K signaling pathways via oxidative stress. 

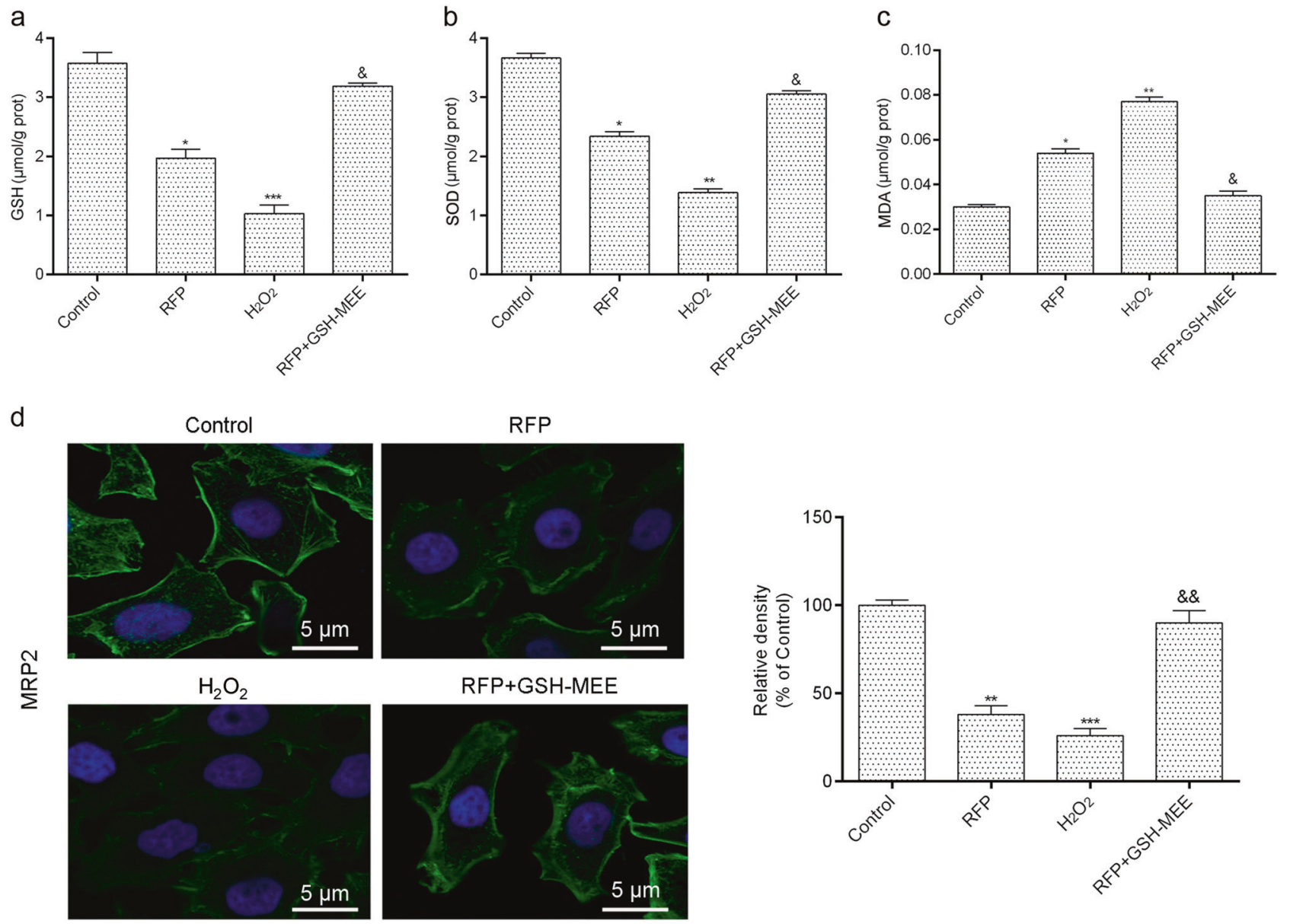

Fig. 2 Rifampicin (RFP) decreased the membrane distribution of multidrug resistance-associated protein 2 (MRP2) by activating oxidative stress in HepG2 cells. a-c Oxidative stress indicators, including glutathione (GSH), superoxide dismutase (SOD), and malondialdehyde (MDA), were measured using microassay kits. d Immunofluorescence staining for MRP2 (green). The nuclei were stained with Hoechst 33342 (blue). Scale bar, $5 \mu \mathrm{m}$. The fluorescence intensity of MRP2 was measured with Image-Pro Plus software. The values are expressed as the mean \pm SD from three independent experiments $(n=3)$. ${ }^{*} P<0.05$, ${ }^{* *} P<0.01$, and ${ }^{* * *} P<0.001$ compared to the control; ${ }^{*} P<0.05$ and ${ }^{\& \&} P<0.01$ vs. the RFP group. Magnification: $\times 400$

RFP caused clathrin-dependent endocytosis of MRP2 via oxidative stress

Compared to the control treatment, treatment with RFP and $\mathrm{H}_{2} \mathrm{O}_{2}$ not only significantly increased the mRNA levels of clathrin and AP2 (Fig. 4a) but also upregulated the protein expression of clathrin and p-AP2; however, these effects were suppressed effectively by GSH-MEE (Fig. 4b). Immunofluorescence analysis also revealed a higher abundance of clathrin and AP2 in the RFP and $\mathrm{H}_{2} \mathrm{O}_{2}$ groups than in the control group of HepG2 cells. However, the fluorescent signals were clearly reduced by the addition of GSH-MEE (Fig. 4C). Co-IP experiments showed that clathrin and AP2 were present in HepG2 cell lysates whether they were treated with RFP or not (input), while these proteins were absent in the IgG group. After capture with the MRP2 antibody, clathrin and AP2 were detected under RFP stimulation but not under control conditions, suggesting that RFP promoted the interactions of MRP2, clathrin, and AP2 (Fig. 4d).

To further explore the effects of clathrin and AP2 on the expression of MRP2 under RFP stimulation, we carried out siRNA experiments. The expression of clathrin and AP2 was successfully downregulated after transfection with si-clathrin and si-AP2, respectively (Fig. $5 a-c$ ). The MRP2-A and MRP2-M levels were significantly higher in both the clathrin siRNA + RFP group and the AP2 siRNA + RFP group than in the siRNA NC + RFP group (Fig. 5a, d, e). Moreover, silencing clathrin and AP2 markedly inhibited the RFP-induced decrease in MRP2-M fluorescence, further illustrating that the RFP-induced decrease in MRP2 was associated with clathrin-dependent endocytosis (Fig. 5f).

Treatment of HepG2 cells with RFP contributed to the ubiquitin-proteasome degradation of MRP2

As shown in Fig. 6a, ubiquitinated MRP2 was detected in immunoprecipitates with MRP2 antibody from HepG2 cells treated with RFP but not in those from control cells. Compared with RFP stress alone, addition of the proteasome inhibitor MG132 along with RFP stress significantly increased the relative expression of MRP2. However, MG132 alone showed no significant effect on MRP2 expression compared with the control treatment of DMSO alone (Fig. 6b). These results implied that the MRP2-M was degraded via ubiquitination after clathrin-dependent endocytosis induced by RFP. Next, we found that RFP significantly upregulated the expression of GP78 but had little effect on the expression of HRD1 and TEB4 (Fig. 6c). Furthermore, knocking out GP78 with siRNA clearly restrained the decrease in MRP2 induced by RFP, suggesting that GP78 was the key E3 ligase in the RFP-induced ubiquitination of MRP2-M (Fig. 6d). 


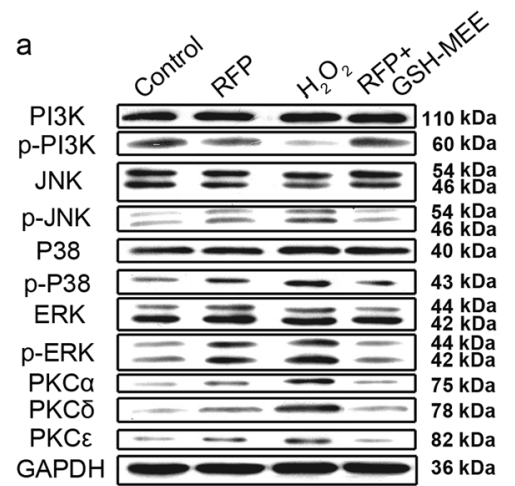

b
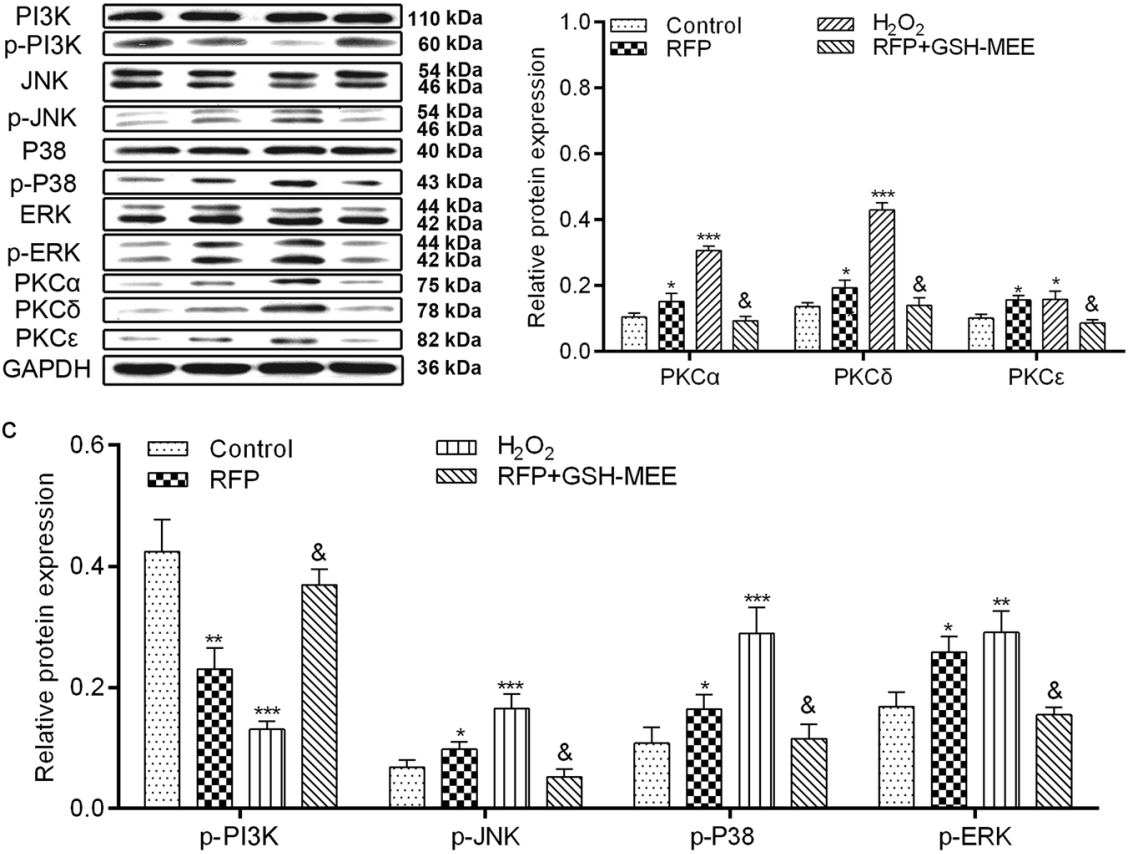

Fig. 3 Rifampicin (RFP) activated the PKC-ERK/JNK/p38 (protein kinase C-extracellular signal-regulated kinase/c-JUN N-terminal kinase/p38) and $\mathrm{PI3K}$ (phosphoinositide 3-kinase) signaling pathways via oxidative stress. a Western blotting was used to detect the expression of PI3K, $\mathrm{PKC} \alpha, \mathrm{PKC} \delta, \mathrm{PKC} \varepsilon, \mathrm{ERK}, \mathrm{JNK}$, and P38, and the phosphorylation of PI3K, ERK, JNK, and P38. $\mathbf{b}$ The relative protein expression of PKC $\alpha$, PKC $\delta$, and PKC $\varepsilon$ and that of (c) phosphorylated (p)-PI3K, p-ERK, p-JNK, and p-P38 were quantified with ImageJ software. The values are expressed as the mean \pm SD from three independent experiments $(n=3) .{ }^{*} P<0.05,{ }^{* *} P<0.01$, and ${ }^{* *} P<0.001$ compared to the control; ${ }^{*} P<0.05$ compared to the RFP group

\section{DISCUSSION}

Antituberculosis drugs are the main cause of acute drug-induced liver injury [17]. The incidence of liver injury caused by RFP is approximately $2 \%$, and the injury is more severe when RFP is combined with isoniazid. Unfortunately, the mechanism has remained unclear until now. Several studies have demonstrated that abnormal expression, altered localization, and dysfunction of hepatobiliary transporter proteins are important in the pathogenesis of intrahepatic cholestasis [18-20]. For example, mutations involving the ATP-binding domains of MRP2 can lead to Dubin-Johnson syndrome, which is characterized by hyperbilirubinemia and elevated bile acid levels. In this study, we found that RFP decreased the membrane localization of MRP2 via clathrindependent endocytosis and ubiquitin-proteasome degradation induced by oxidative stress in HepG2 cells. Our findings provide new ideas and methods for preventing and reducing hepatotoxicity caused by RFP.

MRP2 is encoded by $A B C C 2$ and belongs to the superfamily of ATP-binding cassette transporter proteins [21]. Secretion of GSH and anionic conjugates such as bilirubin and bile acid is the key feature of MRP2. Previous reports have shown that cholestasis induces upregulation of the expression of MRP2 to promote the excretion of bile salts, which has been thought to be an adaptive response to liver damage caused by cholestasis. However, other researchers have reported that hepatic MRP2 expression in cholestatic rodent models is clearly reduced without significant changes in mRNA expression [18, 22]. Similarly, human liver biopsies from inflammation-induced icteric cholestasis (mainly cholestatic alcoholic hepatitis) have been found to display reduced MRP2 immunostaining despite the conservation of MRP2 mRNA levels [23]. In the current study, we found that RFP decreased both MRP2-A and MRP2-M protein levels but did not modify MRP2 mRNA expression in HepG2 cells, indicating that post-transcriptional regulation most likely plays an important role in regulating MRP2 functions (Fig. 1a-c).

It has been demonstrated that oxidative stress is a key feature in most hepatopathies, which can result in cholestasis through actin cytoskeleton disarrangement and further endocytic internalization of bile salt export pump (BSEP) and MRP2 [24]. Sekine and Horie [25] and Sekine et al. [26] reported that ethacrynic acid, which induces acute oxidative stress in the rat liver, reduces $\mathrm{GSH}$, elevates $\mathrm{Ca}^{2+}$, induces NO production, and activates novel PKC in a sequential manner, ultimately leading to MRP2 internalization; the authors reported that these effects were reversibly regulated by the intracellular redox-sensitive balance of PKA/PKC activation. Chronic oxidative stress also decreases hepatic MRP2 protein expression and disrupts the canalicular localization of MRP2 [12]. Here, we discovered that RFP induced oxidative stress in HepG2 cells, decreasing GSH and SOD levels and increasing MDA levels. However, pretreatment of cells with GSH-MEE clearly reversed the changes in GSH, SOD, and MDA levels. Furthermore, replenishment with GSH-MEE successfully inhibited the decrease in the green fluorescence of membrane-distributed MRP2 (Fig. 2a-d). These results clearly demonstrated that RFP decreased MRP2-M levels via oxidative stress in HepG2 cells.

Researchers have considered redox status imbalance to be a common trigger of the signaling pathway leading to MRP2 internalization [27]. Previous studies showed that $\mathrm{Ca}^{2+}$-dependent PKC-MAPK pathways, including the ERK, JNK, and P38-type signaling pathways, participate in hepatocanalicular dysfunction and cholestasis by promoting F-actin rearrangement and further endocytic internalization of canalicular transporters in response to tert-butyl hydroperoxide-induced oxidative stress [28-30]. Moreover, estradiol-17 $\beta-D$-glucuronide (E17G) activates the 


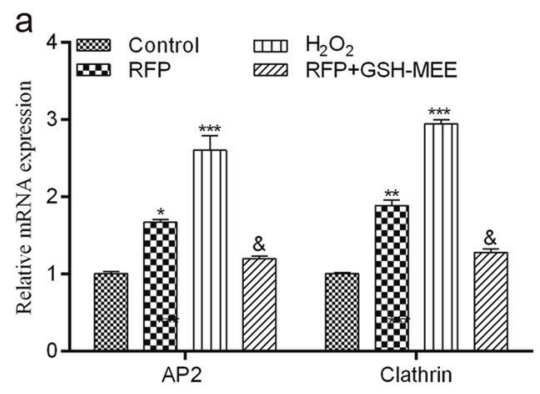

C

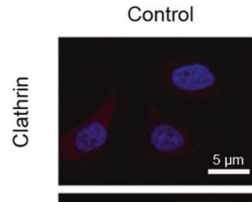

$\frac{N}{4}$
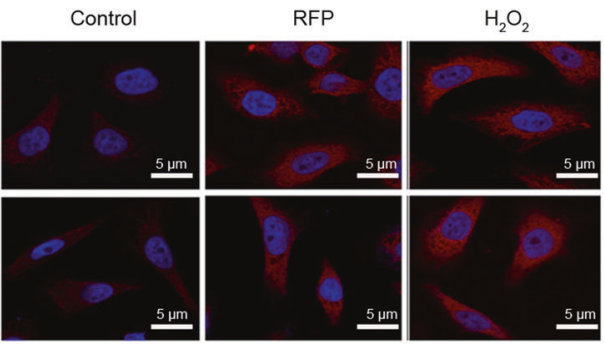

d

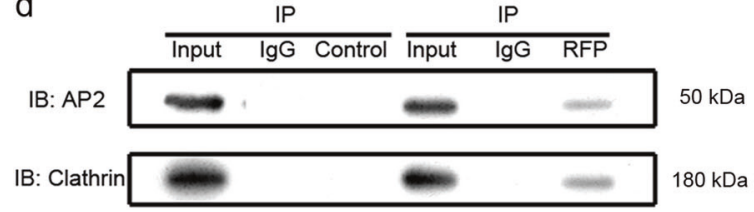

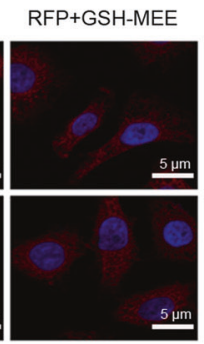

b
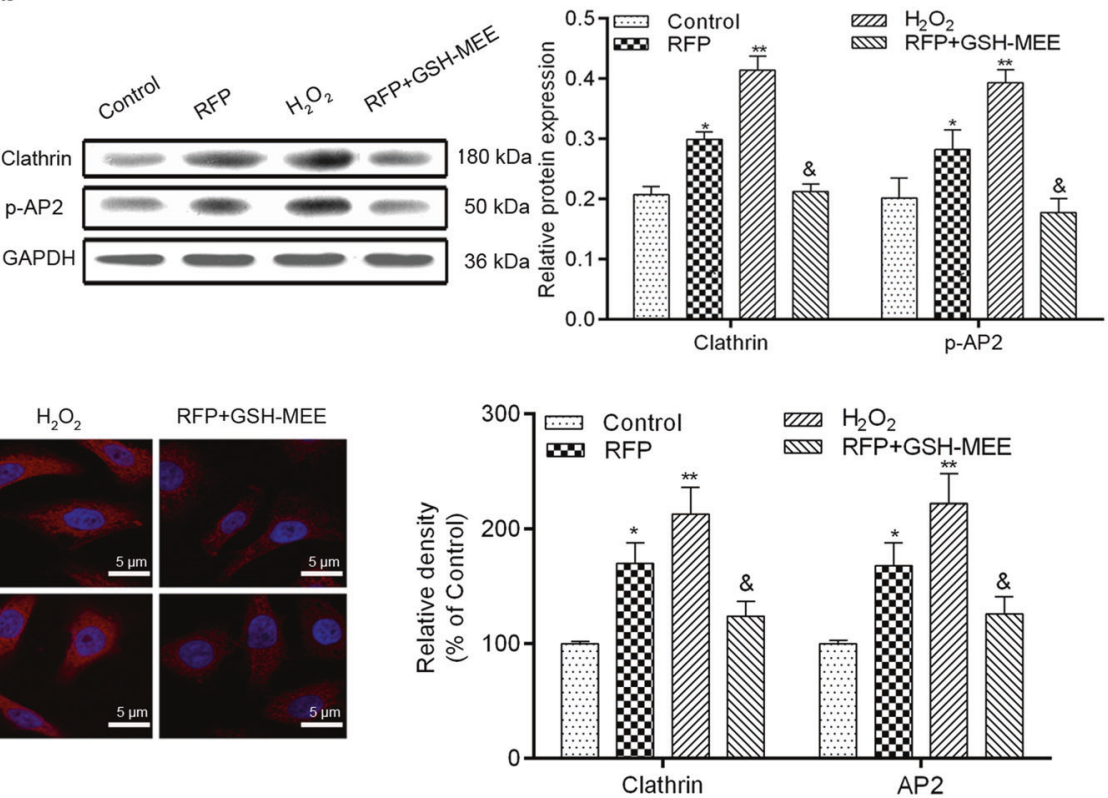

Fig. 4 Rifampicin (RFP) affected the expression of clathrin and adaptor protein 2 (AP2) and the interactions of multidrug resistance-associated protein 2 (MRP2), clathrin and AP2 via oxidative stress. a The messenger RNA (mRNA) levels of clathrin and AP2 were measured by quantitative reverse transcription-polymerase chain reaction (qRT-PCR). b Western blot analysis was used to detect the expression of clathrin and the phosphorylation level of AP2. The relative protein expression levels of clathrin and p-AP2 were quantified with ImageJ. c Immunofluorescence staining for AP2 and clathrin (red). The nuclei were stained with Hoechst 33342 (blue). Scale bar, $5 \mu$ m. d Co-immunoprecipitation (Co-IP) was conducted to explore the interactions of MRP2, clathrin, and AP2. The values are expressed as the mean \pm SD from three independent experiments $(n=3)$. ${ }^{*} P<0.05,{ }^{*} P<0.01$, and ${ }^{* * *} P<0.001$ vs. the control; ${ }^{*} P<0.05$ vs. the RFP group. Magnification: $\times 400$

internalization and sustained intracellular retention of BSEP and MRP2 through the conventional PKC/p38 MAPK and PI3K/ERK1/ 2 signaling pathways, respectively [31]. In the present study, we discovered that RFP-induced oxidative stress was an upstream initiating factor triggering the PKC-ERK/JNK/p38 and PI3K signaling pathways in HepG2 cells, which were inhibited significantly by pretreatment with GSH-MEE (Fig. 3a-c).

Under normal circumstances, the transport and recovery of membrane proteins are maintained in a dynamic state of equilibrium. Proteins are transported from Golgi bodies to the cell surface, where they execute their biological functions, and are then returned to endosomes via endocytosis, in which clathrindependent endocytosis plays a critical role [32]. The PKC-MAPK signaling pathway is necessary for activating clathrin-dependent endocytosis and determines the fates of membrane transporters. AP2, the link between clathrin and transport proteins, is activated by P38 and can mediate the internalization and subsequent degradation of membrane transport proteins [33]. In E17G-treated isolated rat hepatocyte couplets, significant increases in the colocalization of MRP2 with clathrin, AP2, and Rab5 have been reported [34]. Silencing of AP2 with siRNA in rat SCHs completely prevents E17G-induced endocytosis of BSEP and MRP2. We found that the mRNA levels, protein expression, and fluorescence intensity of clathrin and AP2 were markedly upregulated in HepG2 cells treated with RFP and $\mathrm{H}_{2} \mathrm{O}_{2}$, but downregulated in HepG2 cells pretreated with GSH-MEE before being treated with RFP (Fig. 4a-C). Co-IP experiments revealed that RFP increased the interaction of MRP2, clathrin, and AP2 (Fig. 4d). However, knockdown of clathrin and $A P 2$ with siRNAs successfully restrained the reductions in MRP2-M and MRP2-A (Fig. 5a-f). Taken together, the results suggest that RFP-activated oxidative stress may disturb the balance between the transport and recovery of membrane proteins and trigger clathrin-dependent endocytosis via the PKCERK/JNK/p38 and PI3K signaling pathways.

Ubiquitination functions as an internalization signal that sends the modified substrate to endocytic/sorting compartments, after which the substrate is recycled to the plasma membrane or degraded by a proteasome. Ubiquitination is a reversible posttranslational modification involving conjugation of ubiquitin to targeted proteins through sequential reactions mediated by E1, $E 2$, and E3 ubiquitin ligases, which determines their intracellular fates [35]. Among the ubiquitin ligases, E3 ligases, including GP78, HRD1, and TEB4, play pivotal roles in ubiquitination. HRD1 and TEB4 are involved in the degradation of the mutant BSEP in progressive familial intrahepatic cholestasis type II [36]. However, in patients with obstructive cholestasis, GP78 rather than HRD1 and TEB4 participates in MRP2 internalization and degradation activated by liver PKCs, which further leads to Ezrin Thr567 phosphorylation [37]. Aida et al. [13] explained that clathrinmediated endocytosis of BSEP and the degradation of internalized MRP2 facilitated by ubiquitination are responsible for the disappearance of cell surface-resident transporters. We thus investigated whether the RFP-induced decrease in MRP2 was related to ubiquitination. As shown in Fig. $6 a-c$, we discovered that RFP increased the ubiquitin modification and proteasome degradation of MRP2, in which GP78, rather than HDR1 and TEB4, 
a

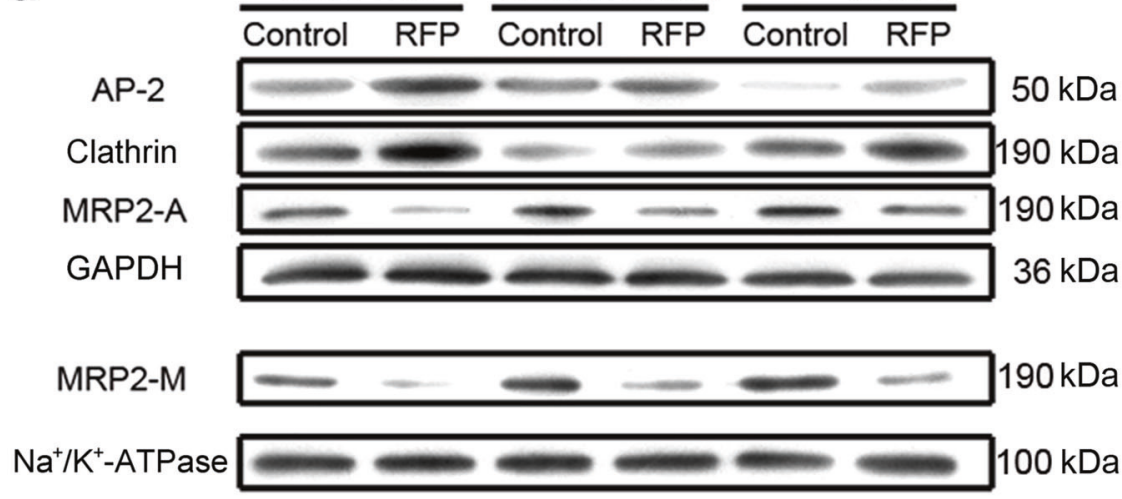

C

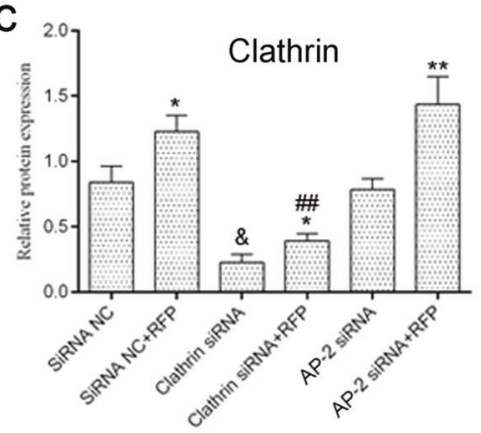

d

f

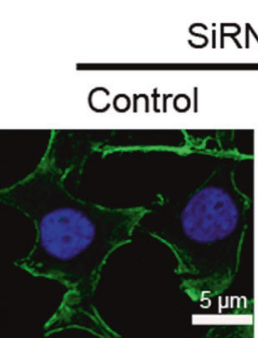

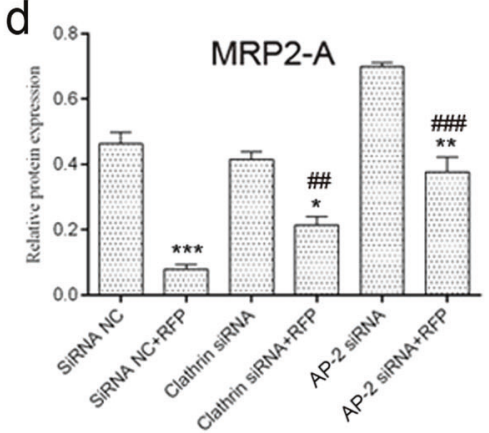

Clathrin siRNA

b

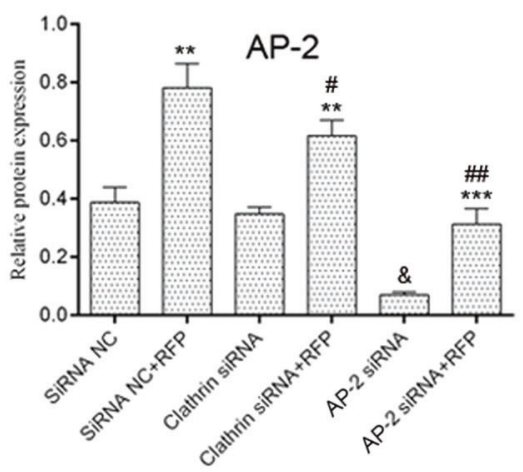

e
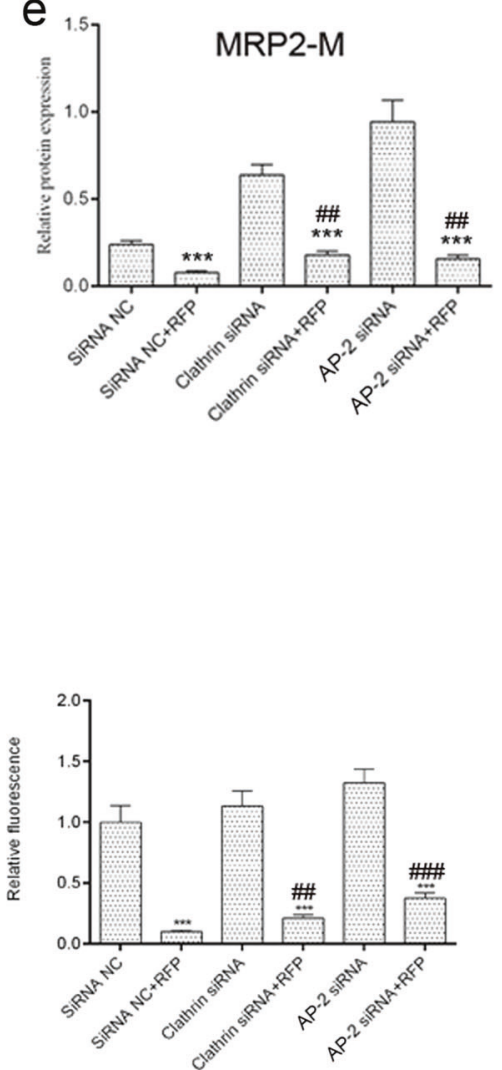

Fig. 5 Rifampicin (RFP) caused clathrin-dependent endocytosis of multidrug resistance-associated protein 2 (MRP2). Western blot analysis was used to measure the transfection efficiency of small interfering RNAs (siRNAs) against clathrin and AP2 (a-c) and the expression of total MRP2 (a, d) and membrane MRP2 (a, e). f The membrane localization of MRP2 was determined with immunofluorescence (green). The nuclei were stained with Hoechst 33342 (blue). Scale bar, $5 \mu \mathrm{m}$. The values are expressed as the mean \pm SD from three independent experiments $(n=3)$. ${ }^{*} P<0.05,{ }^{*} P<0.01$, and ${ }^{* * *} P<0.001$ indicate the results of pairwise comparisons of the siRNA groups. ${ }^{8} P<0.05$ compared to the siRNA NC. ${ }^{\#} P<0.05,{ }^{\# \#} P<0.01$, and ${ }^{\# \# \#} P<0.001$ compared to the siRNA + RFP group. Magnification: $\times 400$

played an important role. Silencing of GP78 with siRNA significantly inhibited the downregulation of MRP2 induced by RFP, which further demonstrated that GP78-mediated ubiquitination determined the fate of MRP2 under RFP stimulation (Fig. 6d).

\section{CONCLUSION}

Our current study suggests that RFP-induced oxidative stress activates the PKC-ERK/JNK/p38 and PI3K signaling pathways, leading to clathrin-dependent endocytosis and ubiquitin-proteasome degradation of MRP2 in HepG2 cells. These findings provide new insights supporting exploration of the mechanisms of RFP-induced cholestasis and suggest that changing the peroxidation states in hepatocytes or inhibiting the ubiquitination of MRP2 may prevent or alleviate the hepatotoxicity caused by RFP. This study was limited to investigating the effect of RFP on MRP2 at the cellular level in vitro. Hence, the relationship between MRP2- and RFP-induced cholestasis should also be explored in vivo. 


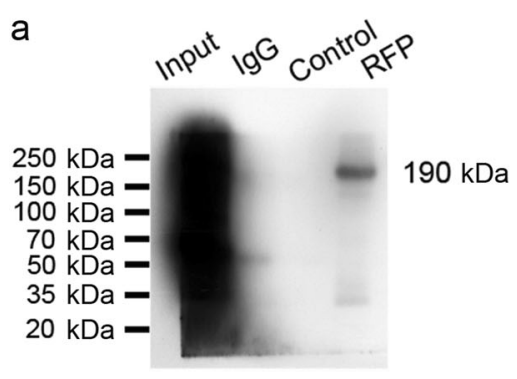

b

C

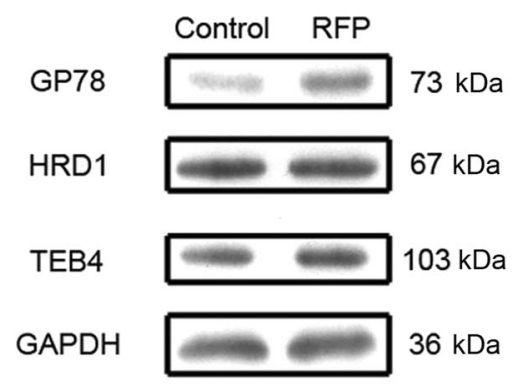

d

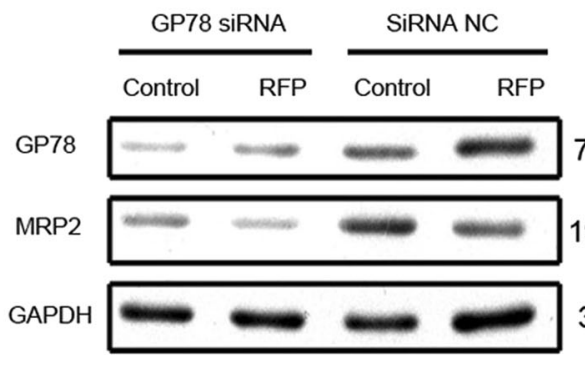

$73 \mathrm{kDa}$

$190 \mathrm{kDa}$

$36 \mathrm{kDa}$
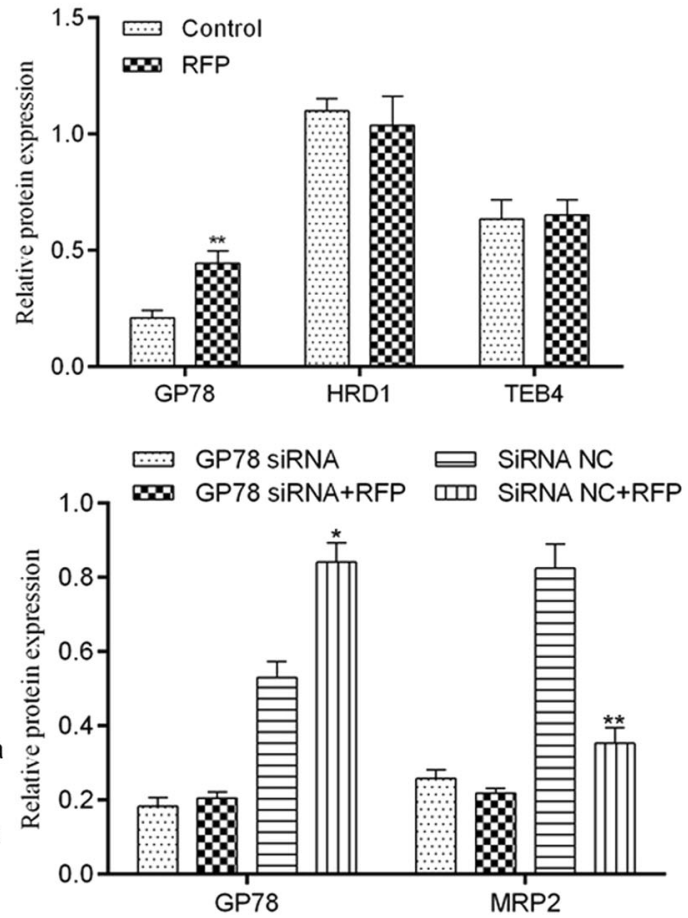

Fig. 6 Treatment of HepG2 cells with rifampicin (RFP) contributed to the ubiquitination of multidrug resistance-associated protein 2 (MRP2). a After treatment with RFP, cell lysates were incubated with MRP2 antibodies or immunoglobulin G (lgG) (as a negative control) to capture the target protein and probed with ubiquitin antibodies. b MRP2 expression was investigated in the presence or absence of MG132 under RFP stress. c The effects of E3 ubiquitin ligases, including GP78, HRD1, and TEB4, were analyzed after treatment with RFP. d The effect of GP78 small interfering RNA (siRNA) on the expression of MRP2 was investigated by Western blotting. The values are expressed as the mean \pm SD from three independent experiments $(n=3)$. ${ }^{*} P<0.05,{ }^{* *} P<0.01$, and ${ }^{* *} P<0.001$ compared to the control. ${ }^{\& \&} P<0.01, \& \& \& P<0.001$ compared to the RFP group. ${ }^{\#} P<0.05$ compared to the MG132 group

\section{ACKNOWLEDGEMENTS}

This work was supported by grants from the National Natural Science Foundation of China (Nos. 81470850 and 30900678) and the Key Project of Science and Technology of Chongqing (CSTC, 2009BB5159).

\section{AUTHOR CONTRIBUTIONS}

All authors contributed to study design and to the analysis and interpretation of the data; BYX and XDT performed the experiments; BYX wrote the paper; and LC reviewed and edited the manuscript. All authors read and approved the final manuscript.

\section{ADDITIONAL INFORMATION}

Competing interests: The authors declare no competing interests.

\section{REFERENCES}

1. Li S, Tan HY, Wang N, Zhang ZJ, Lao L, Wong CW, et al. The role of oxidative stress and antioxidants in liver diseases. Int J Mol Sci. 2015;16:26087-124.
2. Anthérieu S, Bachour-El Azzi P, Dumont J, Abdel-Razzak Z, Guguen-Guillouzo C, Fromenty $B$, et al. Oxidative stress plays a major role in chlorpromazine-induced cholestasis in human HepaRG cells. Hepatology. 2013;57:1518-29.

3. Chowdhury A, Santra A, Bhattacharjee K, Ghatak S, Saha DR, Dhali GK. Mitochondrial oxidative stress and permeability transition in isoniazid and rifampicin induced liver injury in mice. J Hepatol. 2006;45:117-26.

4. Linares V, Alonso V, Albina ML, Bellés $M$, Sirvent JJ, Domingo JL, et al. Lipid peroxidation and antioxidant status in kidney and liver of rats treated with sulfasalazine. Toxicology. 2009;256:152-6.

5. Al-Malki ALSA. Thymoquinone attenuates cisplatin-induced hepatotoxicity via nuclear factor kappa- $\beta$. BMC Complem Alter Med. 2014;14:282.

6. Thongraung WSM, Khwansuwan P, Sariyasuntorn K, Wongsampan S. Evaluation of the physicians' approach to the diagnosis and treatment of patients with antituberculosis drug-induced hepatotoxicity. J Eval Clin Pract. 2012; 18:1119-25.

7. Ichai PSF, Antoun F, Azoulay D, Sebagh M, Antonini TM, Escaut L. et al. Acute liver failure due to antitubercular therapy: Strategy for antitubercular treatment before and after liver transplantation. Liver Transplant. 2010;16:1136-46.

8. Crocenzi FAZA, Boaglio AC, Barosso IR, Sanchez Pozzi EJ, Mottino AD, Roma MG. Localization status of hepatocellular transporters in cholestasis. Front Biosci. 2012;17:1201-18. 
9. Guo YX, Xu XF, Zhang QZ, Li C, Deng Y, Jiang P, et al. The inhibition of hepatic bile acids transporters Ntcp and Bsep is involved in the pathogenesis of isoniazid/ rifampicin-induced hepatotoxicity. Toxicol Mech Methods. 2015;25:382-7.

10. Emina Halilbasic TC, Trauner M. Bile acid transporters and regulatory nuclear receptors in the liver and beyond. J Hepatol. 2013;58:155-68.

11. Paulusma CCKM, Bakker CT, Bosma PJ, van Bokhoven I, van Marle J, Bolder U, et al. Zonal down-regulation and redistribution of the multidrug resistance protein 2 during bile duct ligation in rat liver. Hepatology. 2000;31:684-93.

12. Sekine SMK, Ito K, Kugioka S, Horie T. Sustained intrahepatic glutathione depletion causes proteasomal degradation of multidrug resistance-associated protein 2 in rat liver. Biochim Biophys Acta. 2012;1822:980-7.

13. Aida K, Hayashi $H$, Inamura K, Mizuno T, Sugiyama $Y$. Differential roles of ubiquitination in the degradation mechanism of cell surface-resident bile salt export pump and multidrug resistance-associated protein 2. Mol Pharmacol. 2013; 85:482-91.

14. Sekine SIK, Horie T. Canalicular Mrp2 localization is reversibly regulated by the intracellular redox status. Am J Physiol Gastrointest Liver Physiol. 2008;295: G1035-41.

15. Romagnuolo R, Scipione CA, Boffa MB, Marcovina SM, Seidah NG, Koschinsky ML. Lipoprotein(a) catabolism is regulated by proprotein convertase aubtilisin/kexin type 9 through the low density lipoprotein receptor. J Biol Chem. 2015; 290:11649-62.

16. Wang C, Duan HY, Ma D, Zhou KY, Wang T, Zhang Y, et al. Effect of histone deacetylase Inhibition on the expression of multidrug resistance-associated protein 2 in a human placental trophoblast cell line. Chin Med J. 2017;130:1352.

17. Assosiation CGoHDoDDBoCM. A multicenter survey on hospital inpatients with drug-induced acute liver injury in China. Chin J Dig. 2007;27:439-42.

18. Ruiz ML, Villanueva SSM, Luquita MG, Ikushiro Si, Mottino AD, Catania VA. Beneficial effect of spironolactone administration on ethynylestradiol-induced cholestasis in the rat: involvement of up-regulation of multidrug resistanceassociated protein 2. Drug Metab Dispos. 2007;35:2060-6.

19. Keitel VBM, Warskulat U, Kühlkamp T, Keppler D, Häussinger D, Kubitz R. Expression and localization of hepatobiliary transport proteins in progressive familial intrahepatic cholestasis. Hepatology. 2005;41:1160-72.

20. Keitel VVC, Häussinger D, Kubitz R. Combined mutations of canalicular transporter proteins cause severe intrahepatic cholestasis of pregnancy. Gastroenterology. 2006;131:624-9.

21. Keppler DKJ. Hepatic secretion of conjugated drugs and endogenous substances. Semin Liver Dis. 2000;20:265-72.

22. Halilbasic ECT, Trauner M. Bile acid transporters and regulatory nuclear receptors in the liver and beyond. J Hepatol. 2013;58:155-68.

23. Zollner GFP, Zenz R, Fuchsbichler A, Stumptner C, Kenner L, Ferenci P. et al. Hepatobiliary transporter expression in percutaneous liver biopsies of patients with cholestatic liver diseases. Hepatology. 2001;33:633-46.
24. Basiglio CLTF, Boaglio AC, Arriaga SM, Ochoa JE, Sánchez Pozzi EJ, Mottino AD. et al. Physiological concentrations of unconjugated bilirubin prevent oxidative stress-induced hepatocanalicular dysfunction and cholestasis. Arch Toxicol. 2014;88:501-14.

25. Sekine SIK, Horie T. Oxidative stress and Mrp2 internalization. Free Radic Biol Med. 2006:40:2166-74.

26. Sekine SIK, Saeki J, Horie T. Interaction of Mrp2 with radixin causes reversible canalicular Mrp2 localization induced by intracellular redox status. Biochim Biophys Acta. 2011;1812:1427-34.

27. Sekine S, Ito K, Horie T. Oxidative stress and Mrp2 internalization. Free Radic Biol Med. 2006;40:2166-74.

28. Pérez LMMP, Ahmed-Choudhury J, Elias E, Ochoa JE, Sánchez Pozzi EJ, Coleman $\mathrm{R}$, et al. Oxidative stress induces actin-cytoskeletal and tight-junctional alterations in hepatocytes by a $\mathrm{Ca}^{2+}$-dependent, PKC-mediated mechanism: protective effect of PKA. Free Radic Biol Med. 2006;40:2005-17.

29. Pérez LM, Milkiewicz P, Elias E, Coleman R, Sánchez Pozzi EJ, Roma MG. Oxidative stress induces internalization of the bile salt export pump, bsep, and bile salt secretory failure in isolated rat hepatocyte couplets: a role for protein kinase $C$ and prevention by protein kinase A. Toxicol Sci. 2006;91:150-8.

30. Toledo FDBC, Barosso IR, Boaglio AC, Zucchetti AE, Sánchez Pozzi EJ, Roma MG. Mitogen-activated protein kinases are involved in hepatocanalicular dysfunction and cholestasis induced by oxidative stress. Arch Toxicol. 2017;91:2391-403.

31. Sastre J, Boaglio AC, Zucchetti AE, Toledo FD, Barosso IR, Sánchez Pozzi EJ, et al. ERK $1 / 2$ and p38 MAPKs are complementarily involved inestradiol $17 \beta-D$-glucuronide-induced cholestasis: crosstalk with CPKC and PI3K. PLoS One. 2012;7: e49255.

32. Grandal MVGL, Henriksen L, Andersen MH, Holst MR, Madshus IH, van Deurs B. Differential roles of Grb2 and AP-2 in p38 MAPK- and EGF-induced EGFR internalization. Traffic. 2012;13:576-85.

33. Hayashi $H$, Inamura $K$, Aida $K$, Naoi S, Horikawa R, Nagasaka $H$, et al. AP2 adaptor complex mediates bile salt export pump internalization and modulates its hepatocanalicular expression and transport function. Hepatology. 2012; 55:1889-900.

34. Miszczuk GSBI, Larocca MC, Marrone J, Marinelli RA, Boaglio AC, Sánchez Pozzi EJ. et al. Mechanisms of canalicular transporter endocytosis in the cholestatic rat liver. Biochim Biophys Acta. 2018;1864:1072-85.

35. d'Azzo ABA, Nastasi T. E3 ubiquitin ligases as regulators of membrane protein trafficking and degradation. Traffic. 2005;6:429-41.

36. Wang L, Dong H, Soroka CJ, Wei N, Boyer JL, Hochstrasser M. Degradation of the bile salt export pump at endoplasmic reticulum in progressive familial intrahepatic cholestasis type II. Hepatology. 2008;48:1558-69.

37. Chai J, Cai SY, Liu X, Lian W, Chen S, Zhang L, et al. Canalicular membrane MRP2/ ABCC2 internalization is determined by Ezrin Thr567 phosphorylation in human obstructive cholestasis. J Hepatol. 2015;63:1440-8. 